\title{
Injuries to the inferior alveolar nerve in practices of minor oral surgery
}

\begin{abstract}
This article aims to perform a systematic review of current medical literature on lower alveolar nerve lesions in minor oral surgery. As a methodology, a scan was carried out in the indexing portals of Scientific Electronic Library Online (SciELO), Scopus and Web of Science. A total of 40 articles were found related to this topic. However, 18 presented a low impact factor or lack of indexation in the Sucupira platform (Qualis index) and were excluded, remaining 23 articles that were exhaustively studied and summarized. It was concluded that lesions of the inferior alveolar nerve are often more present in surgical practices, but their risks are not completely absent in other dental practices.
\end{abstract}

Keywords: paraesthesia, neueropraxia, inferior alveolar
Volume II Issue 6 - 2020

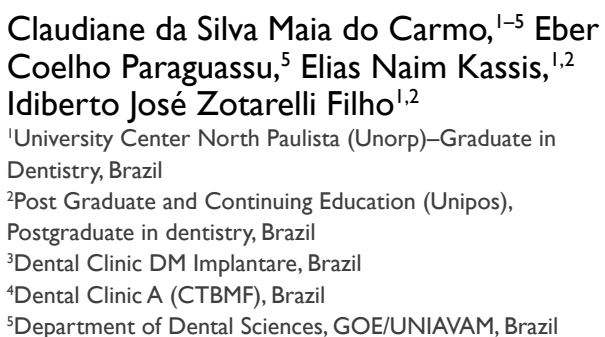

Correspondence: Idiberto José Zotarelli Filho, MSc. Ph.D, Unorp/Unipos, Post graduate and continuing education, Street Ipiranga, 3460, São José do Rio Preto SP, I5020-040, Tel +55( I7) 98166-6537, Email Brazil,

Email scientificresearch@unipos.com.br

Received: December 07, 2020 | Published: December 31, 2020

\section{Introduction}

The trigeminal nerve is one of the most important pairs of cranial nerves in dentistry, and it is indispensable to the dental surgeon to study in an in-depth way, essentially for its capacity of innervation of various structures of the oral cavity and face, such as: teeth, mucosa, muscles, skin and deep facial structures it divides into three large branches and the largest of them is the mandibular nerve. ${ }^{1-3}$

In invasive surgical procedures with proximity to the nerve pathway, we can cause peripheral neuropathic involvement of various natures and degrees, which can lead from small transient lesions to severe and irreparable lesions, which can trigger, paresthesia, dysesthesia, allodynia, hyperalgesia, among others symptoms. Since traumatic injuries are classified as neuropraxias, axonotmeses and neurotmeses. ${ }^{4-7}$

Therefore, the present study aimed to analyze through a systematic review study the injuries to the inferior alveolar nerve in practices of minor oral surgery.

\section{Methods}

\section{Study design}

The present study followed a model of systematic literature review presenting and discussing case series, prospective, retrospective, randomized, double-blind, placebo-controlled trials in humans with a publication time of the last ten years were selected and analyzed, with total of 23 articles, following the rules of PRISMA (Systematic Review)

\section{Search strategy and information sources}

In general, as an example, the search strategy in MEDLINE/ Pubmed, Web of Science, ScienceDirect Journals (Elsevier), Scopus (Elsevier), OneFile (Gale) followed the following steps: - search for mesh terms Paraesthesia. Neueropraxia. Inferior alveolar, and use of the booleans "and" between mesh terms and "or" among historical findings.

\section{Literature review}

The trigeminal nerve is one of the most important pairs of cranial nerves in dentistry, and it is indispensable to the dental surgeon to study in an in-depth way, essentially for its capacity of innervation of various structures of the oral cavity and face, such as: teeth, mucosa, muscles, skin and deep facial structures. It divides into three large branches and the largest of them is the mandibular nerve., ${ }^{1,2}$

The trigeminal nerve is a mixed nerve, with a predominance of its sensory function, being the most important sensory nerve of the head. Its motor root innervates the chewing muscles (masseter, temporal, lateral and medial pterygoid), in addition to the milohyoid muscles, the anterior belly of the digastric muscle, the tensor muscle of the tympanum and the tensile muscle of the palatine veil. ${ }^{3-5}$ Its sensory root leads to general or exteroceptive sensory impulses (touch, pain, temperature) of the following regions: facial skin, ocular conjunctiva, cornea, oral mucosa, part of nasal mucosa, paranasal sinuses, teeth, $2 / 3$ anterior tongue and most of the dura mater. ${ }^{6}$

The mandibular nerve is the only mixed branch of the trigeminal nerve. Its motor branches, for the most part, are called the muscles for which they are intended and their sensory branches are represented by the auricle-temporal, buccal, lingual and inferior alveolar nerves, the latter originating the mental nerve, the incisive nerve and the nerve milo-hyoideo. ${ }^{7-9}$

The inferior alveolar nerve is a sensory nerve, which leads to sensitivity of the lower teeth and vestibular gum of the anterior and lower premolar teeth. It leaves the mandibular canal through the foramen of the mandible, and walks sideways to the medial pterygoid muscle, between the sphenomandibular ligament and the ramus of the 
mandible. In its final course, it is located in the infra-temporal fossa where it joins the posterior trunk of the mandibular nerve. ${ }^{10-13}$

The possibility of injury to the inferior alveolar nerve (NAI) resulting in paresthesia during the surgical removal of impacted lower third molars was amply demonstrated in the literature. The reported incidence of lower alveolar nerve injury after surgical removal of lower third molars ranges from $0.4 \%$ to $8.4 \% .{ }^{14-16}$

The young population today has presented a significant increase of problems related to the region of the third molars, which translate into pain, edema, infection, trismus and difficulty in oral hygiene, due to the spontaneous non-irruption of these teeth. ${ }^{17}$

Removal of an impacted mandibular third molar presents nonsurgical challenges. One of these challenges is the risk of lesions in the peripheral branches of the innervations, which provide sensation to the oral and facial regions. In $95 \%$ of the surgical practices of dentists, one or more patients per year suffered an NAI lesion ( $78 \%$ of the lesions were classified as permanent). ${ }^{18}$

The 3 radiological signs indicate a higher risk of NAI injury, which suggests proximity of the vasculonervoso bundle to the third lower molar. The 3 radiological signs observed were deviation of the mandibular canal, erasure of the dental root, and interruption of the radiopaque line. ${ }^{19}$

The cases of positive radiographic signal, verified in the orthopantomography, can determine a true relation between the apices of the inferior third molar with the mandibular canal. However, this assertion can not be absolute, given that the image obtained in the above-mentioned radiography is of the two-dimensional type, which does not allow registration in the lingual-lingual sense, and often the nerve is more vestibular or lingual to a certain distance from the apexes, giving a false image of positive relation..$^{20}$

The injury to the sensitive branches of the trigeminal nerve (inferior and lingual alveolar), is related to the surgery of removal of third molars. The anatomical position of the lingual nerve with its proximity to adjacent soft tissues puts it at risk during third molar mandibular tooth extractions. The proximity of the third molar roots to the mandibular canal is considered the main risk factor for lower alveolar nerve injury and should be evaluated radiographically with panoramic radiography and computed tomography. ${ }^{21}$

Between 4 and 8 weeks after surgery, 96\% of partial inferior alveolar nerve (NAI) lesions tend to recover. Recovery rates are not influenced by gender and only slightly by age. Some lesions may be permanent or last for more than 6 months and with variable results ranging from mild hypoesthesia to complete anesthesia and exacerbated neuropathic responses, resulting in chronic pain. ${ }^{22}$

The paresthesia of a nerve may have the following causes: mechanical: trauma, compression and / or stretching of the nerve with rupture (partial or total) of its fibers; tissue trauma around nerve fibers; presence of hemorrhages, hematomas and edema around the same, determining the late onset of paresthesia during the first 24-48 hours postoperatively. ${ }^{23}$ Injury to the inferior alveolar nerve may be direct or indirect, the first is caused by injection of anesthetic, surgical instrumentation (osteotomy, odontosection, curettage of the alveoli). Indirect lesions result from the movement of the roots in intimate contact with the walls of the mandibular canal, compression by edema or hematoma. ${ }^{23-26}$

Not all third molars are removed by specialists. There are no recent and constant data available to correlate complications of nerve damage with the surgical practice performed by the dentist. Currently, it is estimated that approximately $50 \%$ of third molars are drawn by generalist dentists in the United States. ${ }^{1}$

Coronectomy or intentional partial odontoctomy is the removal of the crown from the tooth, leaving the root in situ. This technique, when applied for the removal of a third molar or any posterior tooth included in the mandible, is intended to avoid damage to the inferior alveolar nerve. Patel et al. ${ }^{7}$ developed a study histologically evaluating 26 roots in 21 patients who underwent coronectomy and presented painful symptoms. They observed that all roots had vital tissue in the pulp chamber, and there was no evidence of periradicular inflammation. Postoperative painful symptomatology was predominantly related to soft tissue inflammation, which was caused by partially erupted roots or failure in alveolar healing.

Most patients do not resort to treatment and in more than $96 \%$ of cases spontaneous sensory feedback occurs in 24 months. Before starting treatment, it is the professional's duty to first analyze the etiology of paresthesia, because if you are facing an infection, you will probably be giving antibiotics for initial treatment. The use of a low intensity laser has been used in the treatment of long-term sensitive disorders of the inferior alveolar nerve because it is able to react with photosensitive proteins present in different areas of the nervous system, recovering the nervous tissues or affecting the perception of the pain in sensitive nerves. ${ }^{16}$ They showed that in addition to surgery for removal of the third molars, there were other dental practices that could lead to injury to the inferior alveolar nerve. ${ }^{17}$

\section{Conclusion}

In this way, we conclude that lower alveolar nerve lesions are frequently present in surgical practices, but their risks are not completely absent in other dental practices. It was shown as a literary consensus, the need for prior guidance of patients regarding the procedure to be adopted and its risks. Aiming at the patient's good care, during the preoperative consultation, the dental surgeon should guide the patient in the most didactic way possible, adapting the vocabulary to the level of understanding of each patient. Due to the inefficiency of the stipulated treatments for the most severe nervous lesions, the best method of control is still the prevention of lesions by means of study and knowledge of the anatomical structures of the face and the professional experience accompanied by a good planning, adopting an appropriate conduct to reduce risks during the dental procedures employed.

\section{Funding}

None.

\section{Acknowledgments}

None.

\section{Conflicts of interest}

The authors declare that there is no conflict of interest to declare.

\section{References}

1. Piagkou, M, Demesticha T, Piagkos G. Mandibular nerve entrapment in the infratemporal fossa. Surg Radiol Anat. 2011;33(4):291-299.

2. Siqueira R. Peripheral Nerve Injury: A Review. Revista de Neurociência. 2007;15(3).

3. Prades JM. A cadaveric study of a combined trans-mandibular and trans-zygomatic approach to the infratemporal fossa. Surg Radiol Anat. 2003;25(3-4):180-187. 
4. Khan MM, Darwish HH, Zaher WA. Perforation of the inferior alveolar nerve by the maxillary artery: an anatomical study. Br J Oral Maxillofac Surg. 2010;48(8):645-657.

5. Daimi SR, Siddiqui AU, Gill SS. Analysis of foramen ovale with special emphasis on pterygoalar bar and pterygoalar foramen. Folia Morphol. 2011;70(3):149-153.

6. Teixeira LMD, Reher P. Anatomy applied to dentistry. In: Anatomy applied to dentistry: UFMG; 2008.

7. De Castro SV. Anatomia fundamental. Brazil: McGraw-Hill; 1985.

8. Damiani GJ, Céspedes IC. Prevalência de lesão dos nervos alveolar inferior, bucal e lingual em procedimentos operatórios. Odonto. 2007;15(29):50-57.

9. Tay AB, Go WS. Effect of exposed inferior alveolar neurovascular bundle during surgical removal of impacted lower third molars. J Oral Maxillofac Surg. 2004;62(5):592-600.

10. Tachinami, H. et al. Combined preoperative measurement of three inferior alveolar canal factors using computed tomography predicts the risk of inferior alveolar nerve injury during lower third molar extraction. Int J Oral Maxillofac Surg. 2017;46(11):1479-1483.

11. Paraguassu, Éber Coelho, De Cardenas, et al. Sociodemographic characterization of users of total tissue-supported and implant-supported prostheses in the municipality of macapá, Brazil. International Journal of Development Research. 2019;9(2):26081-26084.

12. Da Rosa FM, Escobar CAB, Brusco LC. Parestesia dos nervos alveolar inferior e lingual pós cirurgia de terceiros molares. $R G O$. 2007;5(3):291295.

13. Meyer RA, Bagheri SC. Nerve injuries from mandibular third molar removal. Atlas Oral Maxillofac Surg Clin North Am. 2011;19(1):63-78.

14. Monaco G, Montevecchi M, Bonetti GA, et al. Reliability of panoramic radiography in evaluating the topographic relationship between the mandibular canal and impacted third molars. J Am Dent Assoc. 2004;135(3):312.

15. Sanmarti Garcia G, Valmaseda Castellon E, Gay Escoda C. Does computed tomography prevent inferior alveolar nerve injuries caused by lower third molar removal. J Oral Maxillofac Surg. 2012;70:5-11.

16. Roeder F, Wachtlin D, Schulze R. Necessity of 3D visualization for the removal of lower wisdom teeth: required sample size to prove noninferiority of panoramic radiography compared to CBCT. Clin Oral Investig. 2012;16:699-706.
17. Neves FS, de Almeida SM, Bo'scolo FN, et al. Risk assessment of inferior alveolar neurovascular bundle by multidetector computed tomography in extractions of third molars. Surg Radiol Anat. 2012;34(7):619-624.

18. Guerrero ME, Nackaerts O, Beinsberger J, et al. Inferior alveolar nerve sensory disturbance after impacted mandibular third molar evaluation using cone beam computed tomography and panoramic radiography: a pilot study. J Oral Maxillofac Surg. 2012;70:2264-70.

19. Nakayama K, Nonoyama M, Takaki Y, et al. Assessment of the relationship between impacted mandibular third molars and inferior alveolar nerve with dental 3-dimensional computed tomography. J Oral Maxillofac Surg. 2009;67(12):2587-2591.

20. Éber Coelho Paraguassu, Anneli Celis Mercedes de Cardenas, Marina Nolli Bittencourt, et al. Quality of life and satisfaction of users of total tissue-supported and implant-supported prostheses in the municipality of macapá, Brazil. International Journal of Development Research. 2019;9(2):26007-26011.

21. Hasegawa T, Ri T, Shigeta T, et al. Risk factors associated with inferior alveolar nerve injury after extraction of the mandibular third molar-a comparative study of preoperative images by panoramic radiography and computed tomography. Int J Oral Maxillofac Surg. 2013;42(7):843851

22. Ueda M, Nakamori K, Shiratori $\mathrm{K}$, et al. Clinical significance of computed tomographic assessment and anatomic features of the inferior alveolar canal asrisk factorsfor injury of the inferior alveolar nerve at third molar surgery. . 2012;70(3):514-520.

23. Shiratori K, Nakamori K, Shiratori K, et al. Clinical significance of computed tomographic assessment and anatomic features of the inferior alveolar canal asrisk factorsfor injury of the inferior alveolar nerve at third molar surgery. J Oral Maxillofac Surg. 2012;70(3):514-520.

24. Nakamori K, Fujiwara K, Miyazaki A, et al. Clinical assessment of the relationship between the third molar and the inferior alveolar canal using panoramic images and computed tomography. J Oral Maxillofac Surg. 2008;66(11):2308-2313.

25. Nakamori K, Tomihara K, Noguchi M. Clinical significance of computed tomography assessment for third molar surgery. World $J$ Radiol. 2014;6(7):417-423.

26. Tolstunov L. The quest for causes of inferior alveolar nerve injury after extraction of mandibular third molars. J Oral Maxillofac Surg. 2014;72(9):1644-1646. 